\title{
Genomic and proteomic investigation of preeclampsia
}

\author{
HAYRETTIN SAHIN $^{1}$, TUBA GUNEL ${ }^{2}$, ALI BENIAN ${ }^{1}$, EVREN ONAY UCAR $^{2}$, \\ ONUR GURALP $^{1}$ and AYDINLI KILIC ${ }^{3}$ \\ ${ }^{1}$ Department of Obstetrics and Gynecology, Cerrahpasa Medical Faculty, Istanbul University, Istanbul 34098; \\ ${ }^{2}$ Department of Molecular Biology and Genetics, Faculty of Science, Istanbul University, Istanbul 34134; \\ ${ }^{3}$ Medicus Health Center, Istanbul 34365, Turkey
}

Received July 14, 2014; Accepted April 28, 2015

DOI: $10.3892 / e t m .2015 .2509$

\begin{abstract}
The aim of this study was to use proteomic and transcriptomic approaches to examine differences in protein and gene expression in maternal plasma between normal and preeclamptic pregnancies. Preeclampsia and control groups were compared with respect to the expression of $\mathrm{CD} 34$ and CD133 genes by quantitative polymerase chain reaction (qPCR) and heat shock protein (Hsp) 27 and 70 by western blotting in blood samples from the pregnant women. Blood samples were obtained at gestational week 12-14 from 65 healthy pregnant women. Fetal DNA was isolated from the maternal blood and CD34 and CD133 were amplified by qPCR. Western blot analysis was used to examine the expression levels of Hsp27 and Hsp70 proteins. The analysis of CD133 by qPCR was unsuccessful in 7 women as the levels of fetal DNA were in the collected maternal blood samples were insufficient. Measurements of CD34 and CD133 were performed in 57 and 50 women, respectively. Preeclampsia developed in $6(10.5 \%)$ of 57 women. qPCR results of 8 healthy pregnant women were used for the calibration of CD34 and CD133 levels, and the results for the remaining women were compared with the calibration values. CD34 expression was decreased in 30 (52.6\%) and increased in 27 (47.4\%) of 57 women. CD133 expression was decreased in $14(28 \%)$ and increased in $36(72 \%)$ of 50 women. CD34 expression was increased in 2 (33\%) and 25 (49\%) and decreased in $4(66 \%)$ and $25(51 \%)$ women with and without preeclampsia, respectively $(\mathrm{P}=0.467)$. $\mathrm{CD} 133$ expression was increased in $4(66 \%)$ and $32(72 \%)$; and decreased in $2(33 \%)$ and $12(28 \%)$ women with and without preeclampsia, respectively $(\mathrm{P}=0.756)$. Western blotting showed that the expression of Hsp27 and Hsp70 in the maternal serum of the preeclampsia group was significantly higher than that in the normal pregnancy group. CD34 and CD133 were found to be inadequate
\end{abstract}

Correspondence to: Dr Tuba Gunel, Department of Molecular Biology and Genetics, Faculty of Science, Istanbul University, Vezneciler Street, Istanbul 34134, Turkey

E-mail: gunel@istanbul.edu.tr

Key words: non-invasive prenatal diagnosis, preeclampsia, cell-free fetal DNA for use in the prediction of preeclampsia. However, it is noteworthy that CD133 levels were increased in 66 and $72 \%$ of women with and without preeclampsia, respectively. Hsps are expressed under various pathological conditions. These results suggest that conditions of oxidative stress increased the Hsp27 and Hsp70 protein levels.

\section{Introduction}

Preeclampsia is major cause of maternal morbidity and mortality that is characterized by the development of hypertension and proteinuria after 20 weeks of gestation (1) and complex disorders, including placental and maternal components (2). Recent advances in genotyping technology are likely to facilitate genome-wide association studies of preeclampsia. Nucleated fetal erythrocytes, trophoblasts, leukocytes and even fetal progenitors can cross the placenta (3). Gussin et al (4) have shown that endothelial progenitor cells (EPCs) are present in peripheral blood in the second trimester. Moreover, Sugawara et al (5) demonstrated that the number of EPCs increases in the second and third trimesters. Buemi et al (6) observed that the number of isolated EPCs gradually increases every trimester.

Free fetal DNA particles in maternal blood are promising as an important means for the diagnosis of genetic abnormalities of the fetus without invasive procedures. Quantitative changes of cell-free fetal DNA in maternal blood as an indicator for impending preeclampsia have been reported in numerous studies. When the cell-free fetal DNA levels of 30 preeclamptic patients were compared with those of healthy pregnant women, an average 3.06-fold increase was observed (7).

CD34 is a transmembrane protein that is expressed in 1-4\% of human bone marrow cells. These cells include pluripotent hematopoietic stem cells and progenitors of each cell line (8). In addition, CD34 is expressed in the vascular endothelium of various organs. Fetal $\mathrm{CD} 34^{+}$cells are detected in maternal blood during pregnancy (9). Moreover, fetal CD34+ cells are known to persist in the maternal circulation even 27 years after delivery (10). CD133, also known as prominin-1, is a protein expressed in hematopoietic stem and progenitor cells; however, its function is not fully understood $(11,12)$.

In the last 20 years, antibodies against CD34 (13) and CD133 $(11,12)$ have been widely used as markers for hematopoietic and progenitor cells (14), and it has been shown 
that $\mathrm{CD}_{34}{ }^{+}$and $\mathrm{CD}_{133}{ }^{+}$progenitor cells increase in normal pregnancy and decrease in preeclampsia.

Adhesion molecules, including the EPC marker CD $34^{+}$, have been found in allogenic and xenogenic aortic valve prostheses (15). Therefore, in end-stage calcified aortic stenosis, the presence of EPCs appears to be a biological hallmark, among others such as inflammatory infiltration, heat shock protein (Hsp) homologues and neoangiogenesis (16). Further advances are expected from proteomic research. Comparisons of protein patterns between healthy patients and those with disease have been increasingly conducted in recent years to discover markers of disease. Hsps are overexpressed in all organisms to protect them from environmental stresses such as heat, oxidative stress and ischemia (17). Hsp27 and Hsp70 are well-known stress response proteins. Studies have shown that Hsp70 levels in pregnant women are significantly higher than those in healthy pregnant women $(18,19)$. It is also known that placental levels of Hsp27 in normal patients are lower than those in patients with preeclampsia (20).

In the light of this information, the aim of the present study was to evaluate cell free fetal DNA encoding CD34 and CD133 in the maternal blood from randomly selected healthy pregnant women in gestational week (GW) 12-14. During the follow-up, the free fetal DNA levels of women who did and did not develop preeclampsia were compared in order to investigate the possible predictive role of CD34 and CD133 in preeclampsia. Hsp27 and Hsp70 heat shock protein levels were also compared between the two groups of patients.

\section{Materials and methods}

Study design and sample collection. This prospective study was conducted in 80 consecutive healthy pregnant women who were admitted to the Obstetrics and Gynecology Clinic, Cerrahpasa Medical Faculty, Istanbul University (Istanbul, Turkey). This study was approved by the Cerrahpasa Medical Faculty Ethics Committee. Informed consent was taken from every woman enrolled in the study. Multiple pregnancies and any systemic diseases complicating pregnancy were excluded. In order to increase the possibility of acquiring more preeclamptic women, and also to prevent possible bias, only first pregnancies were included.

During follow-up 8 women developed gestational diabetes; 3 women developed pregnancy-induced hypertension; 2 women developed pregnancy-induced cholestasis; 1 woman had a termination of pregnancy at GW 19 and 1 woman withdrew from follow-up. The studied was completed with 65 women. Six of the 65 women (9.2\%) developed preeclampsia.

Blood samples were taken from the pregnant women in GW 12-14. Prior to any procedure, $5 \mathrm{ml}$ maternal venous blood was sampled and collected in an ethylenediaminetetraacetic tube. Immediately after sampling, the plasma fraction was obtained by centrifugation of the blood sample at $10,000 \mathrm{x} \mathrm{g}, 4^{\circ} \mathrm{C}$ for $10 \mathrm{~min}$ and $500 \mu \mathrm{l}$ from the supernatant layer was used. The diagnosis of preeclampsia was made by measurement of $\geq 140 \mathrm{mmHg}$ systolic blood pressure and $\geq 90 \mathrm{mmHg}$ diastolic pressure at least twice, with $\mathrm{a} \geq 6 \mathrm{~h}$ interval between measurements; and $\geq 300 \mathrm{mg} / 24 \mathrm{~h}$ proteinuria or $1^{+}$proteinuria in the dipstick test after GW 20 in a woman who did not previously have hypertension or a renal disorder.
Table I. Primer sequences used for qPCR.

\begin{tabular}{ll}
\hline Gene & \multicolumn{1}{c}{ Sequence $\left(5^{\prime}-3^{\prime}\right)$} \\
\hline CD34 & F: CCTGATGAATCGCCGCAGCTGGAGC \\
& R: CCTGGCCTCCACCGTTTT \\
GAPDH & F: GAAGGTGAAGGTCGGAGT \\
& R: GAAGATGGTGATGGGATTTC \\
CD133 & F: GGACCCATTGGCATTCTC \\
& R: CAGGACACAGCATAGAATAATC \\
\hline
\end{tabular}

qPCR, quantitative polymerase chain reaction; $F$, forward; $R$, reverse.

Plasma fractions were processed in the Molecular Diagnosis Laboratory, Department of Molecular Biology and Genetics, Cerrahpasa Medical Faculty, Istanbul University.

Maternal plasma fetal DNA processing and quantitation. A 150- $\mu 1$ sample of plasma was used for each isolate. An innuPREP virus DNA/RNA kit (Analytik Jena/Biometra, Germany) was used for the isolation of fetal DNA from the maternal blood according to the instructions of the manufacturer.

Gene sequences encoding $\mathrm{CD}_{34}{ }^{+}$and $\mathrm{CD}_{133^{+}}$surface antigens were used in order to quantitatively measure the fetal DNA in the maternal blood; and a gene sequence encoding glyceraldehyde phosphate dehydrogenase (GAPDH), which is located in the autosomal region, was used as an indicator of total DNA in the maternal blood since its expression is constant at all times.

The primer sequences used for quantitative polymerase chain reaction (qPCR) are shown in Table I. Platinum SYBR Green qPCR SuperMix-UDG (Invitrogen Life Technologies, Carlsbad, CA, USA) was used for the amplification and detection of DNA and a final primer concentration of $250 \mathrm{nM}$ was used as template for the isolation of CD34 and CD133. Reactions were performed in a total volume of $25 \mu \mathrm{l}$.

The qPCR consisted of 1 cycle of denaturation for $10 \mathrm{~min}$ at $95^{\circ} \mathrm{C}$ total followed by 50 cycles comprising denaturation for $30 \mathrm{sec}$ at $95^{\circ} \mathrm{C}$, binding for $1 \mathrm{~min}$ in $56^{\circ} \mathrm{C}$ and elongation for $1 \mathrm{~min}$ at $72^{\circ} \mathrm{C}$. The qPCR was performed using a Stratagene Mx3005P qPCR system (Agilent Technologies, Santa Clara, CA, USA). qPCR results of 8 healthy pregnant women were used for the calibration of CD34 and CD133 levels, and the results for the remaining women were compared with the calibration values. Patients with results above and below the calibration values for CD34 and CD133 genes were identified for the preeclamptic and control groups. As the qPCR efficiency was assumed to be $100 \%$, a value of 2 was used during calculation, instead of the 1.93 coefficient that is indicated in the original formula. qPCR efficiencies were calculated using slopes obtained from LightCycler software (Roche Diagnostics, Basel, Switzerland). The corresponding qPCR efficiency (E) of one cycle in the exponential phase was calculated according to the equation: $\mathrm{E}=10^{-1} /$ slope.

Sodium dodecyl sulfate-polyacrylamide gel electrophoresis (SDS-PAGE) and western blot analyses. Anti-Hsp27 mono- 
Table II. Comparison of CD34 expression between the preeclamptic and non-preeclamptic groups.

\begin{tabular}{lccc}
\hline Expression $^{\mathrm{a}}$ & Preeclamptic $(\mathrm{n}=6)$ & Non-preeclamptic $(\mathrm{n}=51)$ & $\mathrm{P}-\mathrm{value}$ \\
\hline Decreased & 4 & 26 & 0.467 \\
Increased & 2 & 25 &
\end{tabular}

${ }^{a}$ Compared with a calibration value from 8 healthy pregnant women. ${ }^{b}$ Determined by $\chi^{2}$ test; $\mathrm{P}<0.05$ is significant.

Table III. Comparison of CD133 expression between the preeclamptic and non-preeclamptic groups.

\begin{tabular}{lccc}
\hline Expression $^{\mathrm{a}}$ & Preeclamptic $(\mathrm{n}=6)$ & Non-preeclamptic $(\mathrm{n}=51)^{\mathrm{P}-\mathrm{value}^{\mathrm{b}}}$ \\
\hline Decreased & 2 & 12 & 0.756 \\
Increased & 4 & 32 & \\
\hline
\end{tabular}

${ }^{a}$ Compared with a calibration value from 8 healthy pregnant women. ${ }^{b}$ Determined by $\chi^{2}$ test; $\mathrm{P}<0.05$ is significant.

clonal antibody (SPA-800), anti-Hsp70 monoclonal antibody (SPA-810) and horseradish peroxide (HRP)-conjugated goat anti-mouse IgG secondary antibody (SAB-100) were purchased from Stressgen Biotechnologies Corporation (Victoria, Canada). The ECL Plus Western Blotting detection reagent was purchased from Amersham (GE Healthcare, Piscataway, NJ, USA). The bicinchoninic acid (BCA) assay kit was obtained from Pierce (Thermo Fisher Scientific, Rockford, IL, USA). The other reagents were obtained from Sigma-Aldrich (Chicago, IL, USA).

The protein concentration of each sample was determined using the BCA kit. Bovine serum albumin was used as a standard. For SDS-PAGE and western blot analysis, samples were denatured in sample buffer containing $25 \mathrm{mM}$ Tris- $\mathrm{HCl}$ (pH 6.8), 2\% SDS, $10 \%$ glycerol, $10 \%$ 2-mercaptoethanol and $0.002 \%$ bromphenol blue and heating to boiling temperature for $3 \mathrm{~min}$. Equal amounts of proteins (100 $\mu \mathrm{g} /$ well) were analyzed by $10 \%$ SDS-PAGE. After electrophoresis, proteins were transferred onto polyvinylidene difluoride membranes using Bio-Rad Semi-Dry transfer apparatus (Bio-Rad Laboratories, Inc., Hercules, CA, USA). Membranes were blocked with 5\% non-fat dry milk (in Tris-buffered saline and Tween 20) at room temperature for $1 \mathrm{~h}$, and then incubated with the primary antibodies anti-Hsp70 (dilution, 1:1,000) and anti-Hsp27 (dilution, 1:500) for $1 \mathrm{~h}$ at room temperature. The membrane was then incubated with HRP-conjugated goat anti-mouse secondary antibodies at a dilution of 1:5,000 for $1 \mathrm{~h}$ at room temperature. Protein bands were detected by incubating the membranes with ECL plus. Protein levels were quantitatively evaluated with ChemiDoc MP and ImageLab 4.0.1 software (Bio-Rad).

Statistical analysis. Data are presented as means, standard deviations and rates. Statistical analysis was performed using the t-test and $\chi^{2}$ test. Correlation analyses were performed with the Spearman correlation test. $\mathrm{P}<0.05$ was considered statistically significant. All statistical analyses were performed using the Statistical Package for the Social Sciences (SPSS ${ }^{\circledR}$ ) software version 16.0 (SPSS, Inc., Chicago, IL, USA)

\section{Results}

Patient characteristics. At the initiation of the study, 80 women were enrolled. Following a termination of pregnancy for one woman and the withdrawal of one patient from follow-up, 78 women remained. The incidence of preeclampsia in these women was $7 \%$ (6/78).

As mentioned in Materials and methods, 13 more women were excluded because of pregnancy complications. A total of 65 pregnant women completed the study and fulfilled the inclusion criteria. Samples from 8 women were used for the calibration of CD34 and CD133 levels and, therefore, excluded from the statistical calculations. During CD133 evaluation, the qPCR process was unsuccessful in 7 cases. Statistical evaluations of CD34 and CD133 were performed for 57 and 50 women, respectively.

The mean age of the 57 women for whom qPCR measurements were analyzed was $29.4 \pm 4.8$ years. Among these 57 women, $6(10.5 \%)$ developed preeclampsia. There was no significant difference in the mean age of the preeclamptic and non-preeclamptic women $(29.6 \pm 4.6$ vs. $28.1 \pm 6.1$ years, respectively; $\mathrm{P}=0.485$ )

CD34 and CD133 expression. Expression levels of CD34 and CD133 in the two groups were compared with the calibration results from 8 healthy pregnant women. Among 57 women, CD34 expression was decreased in $30(52.6 \%)$ and increased in $27(47.4 \%)$ cases. Among 50 women, CD133 expression was decreased in 14 (28\%) and increased in 36 (72\%) cases.

CD34 expression was compared between the preeclamptic $(n=6)$ and non-preeclamptic $(n=51)$ groups. In the preeclamptic group, CD34 expression was increased in 2 (33.3\%) and decreased in 4 (66.6\%) women. In the non-preeclamptic group, CD34 expression was increased in $25(49 \%)$ and decreased in $26(51 \%)$ women $(\mathrm{P}=0.467$; Table II).

CD133 expression was compared between the preeclamptic $(n=6)$ and non-preeclamptic $(n=44)$ groups. In the preeclamptic group, CD133 expression was increased in 4 (66.6\%) and decreased in $2(33.3 \%)$ women. In the non-preeclamptic group, 


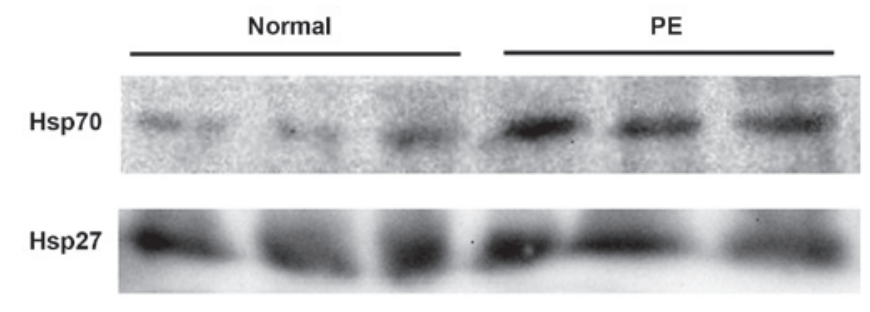

Figure 1. Western blot analysis of Hsp70 and Hsp27 in normal and preeclamptic (PE) women. For each lane, $100 \mu \mathrm{g}$ serum protein was loaded and subjected to SDS-PAGE followed by western blot analysis as described in Materials and methods. Hsp, heat shock protein; SDS-PAGE, sodium dodecyl sulfate-polyacrylamide gel electrophoresis.

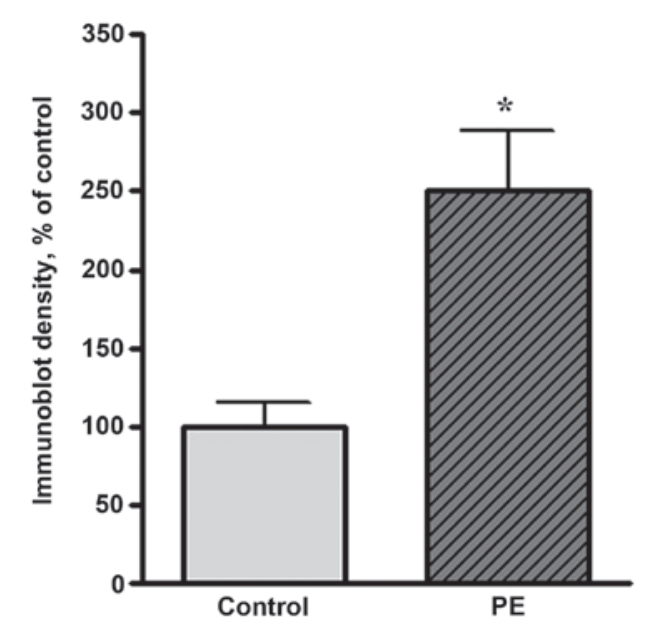

Figure 2. Quantitative results of western blot analysis of Hsp70 in normal and preeclamptic $(\mathrm{PE})$ women. The $\mathrm{y}$-axis indicates the band intensities defined by the number of pixels under each peak $(\mathrm{n}=6 ; * \mathrm{P}<0.05$ compared with the control group). Hsp, heat shock protein.

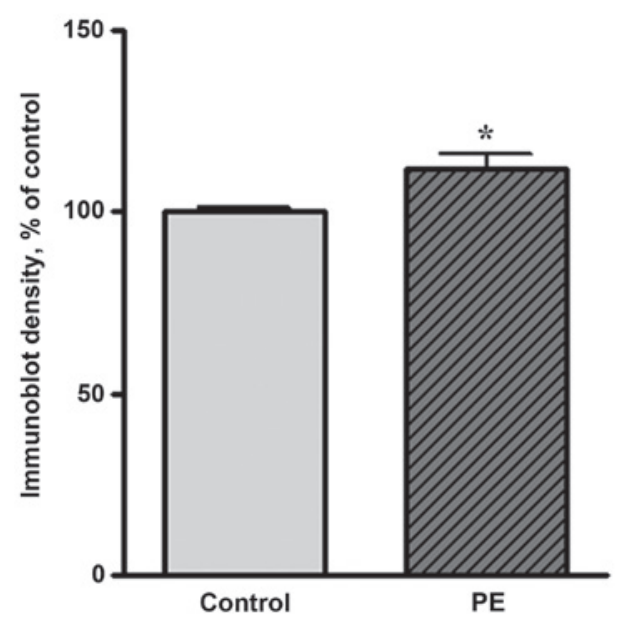

Figure 3. Quantitative results of western blot analysis of Hsp27 in normal and preeclamptic women. The $y$-axis indicates the band intensities defined by the number of pixels under each peak. $\left(\mathrm{n}=6,{ }^{*} \mathrm{P}<0.05\right.$ compared with the control group). Hsp, heat shock protein.

CD133 expression was increased in $32(72 \%)$ and decreased in 12 (28\%) women $(\mathrm{P}=0.756$; Table III).

Spearman's correlation analysis showed that there was no correlation between CD34 and CD133 ( $\mathrm{P}=0.122$; $r=0.219)$.
Hsp27 and Hsp70 expression. Western blot analysis showed that the expression levels of Hsp27 and Hsp70 were markedly increased in preeclamptic women compared with healthy pregnant women. A representative western blot result is shown in Fig. 1. The level of Hsp70 in preeclamptic women was significantly increased compared with that in healthy pregnant women (by $150 \%, \mathrm{P}<0.05$; Fig. 2). The Hsp27 level was increased by $11 \%$ of the control level in preeclamptic women (Fig. 3).

\section{Discussion}

In recent studies concerning the pathophysiology of preeclampsia, the idea that an altered balance of angiogenic [vascular endothelial growth factor (VEGF) and placental growth factor (PlGF)] and antiangiogenic [soluble fms-like tyrosine kinase-1 (sFlt-1) and soluble endoglin (sEng)] factors may play role in the pathogenesis of preeclampsia gained importance. According to this theory, the antiangiogenic factor sFlt-1 is released from the hypoxic placenta. VEGF interacts with its receptor on cell membranes where it exerts its effects via Flt-1; VEGF is a strong angiogenic factor and causes vasodilatation. sFlt-1 is the soluble and, therefore, detectable form of Flt-1, and is found in maternal serum. sFlt-1 is an antiangiogenic factor that shows antagonistic effects against VEGF and PIGF by binding to them in the serum (21).

Eng is the co-receptor of transforming growth factor (TGF)- $\beta 1$ and TGF- $\beta 3$, and is widely expressed in vascular endothelium and syncytiotrophoblasts. The TGF- $\beta 1$ signaling pathway contains CD34 and CD133. The soluble form of Eng, sEng, is an antiangiogenic factor and inhibits the TGF- $\beta 1$ pathway $(21,22)$.

The present study focused on two different genes that have the potential to be markers for preeclampsia. CD34-expressing cells are precursors of hematopoietic and endothelial cell lines and are known to be regulators of angiogenesis $(23,24)$. The other marker, CD133, was first defined in the neuroepithelial stem cells of mice (25); its function is not well established, although it is considered to play a role in angiogenesis. In the human body, CD133 is known to be an antigenic marker expressed in hematopoietic stem and progenitor cells $(11,12)$.

In preeclamptic women, free fetal DNA levels increase 3-fold compared with those in healthy pregnant women (7,12,26-28). Zhong et al (29) suggested that DNA levels were higher in early-onset $(<34 \mathrm{GW})$ preeclampsia compared with late onset $(>34 \mathrm{GW})$ preeclampsia and healthy pregnant women; this finding was attributed to placental damage. Sifakis et al (30) evaluated the serum samples of pregnant women at GWs 11-13 and showed that the levels of fetal DNA fragments were significantly higher in early onset compared with late onset preeclampsia. In the present study, the aim was to go a step further by evaluating the potential predictive value of CD34 and CD133. Therefore, only healthy pregnant women at GW 12-14 were enrolled.

The amount of free fetal DNA in the maternal serum of healthy pregnant women has been shown to increase with increasing gestational age (31). Moreover, the ratio of fetal DNA to total free DNA has been shown to increase every trimester, although total free DNA remains unaffected by gestational age (32). 
In the present study, in the non-preeclamptic group, also denoted as the healthy group, CD34 was decreased in $26(51 \%)$ and increased in 25 (49\%) of 51 women; CD133 was decreased in $12(28 \%)$ and increased in $32(72 \%)$ of 44 women. In the preeclamptic group, CD34 expression was increased in $2(33.3 \%)$ and decreased in $4(66.6 \%)$; whereas CD133 expression was increased in $4(66.6 \%)$ and decreased in $2(33.3 \%)$. There was no significant difference between preeclamptic and non-preeclamptic women, neither in CD34 nor in CD133 expression. Spearman's correlation analysis showed that there was no correlation between CD34 and CD133.

Luppi et al (14) detected that circulating $\mathrm{CD}^{+} 4^{+}$and $\mathrm{CD}_{133}{ }^{+}$progenitor cells were increased in normal pregnancy and decreased in preeclamptic women. By contrast, Matsubara et al (33) showed that the number of $\mathrm{CD} 34^{+}, \mathrm{CD} 133^{+}$ and $\mathrm{VEGF}^{+}$cells in the maternal circulation increased with increasing gestational age, and that there was no significant difference in the number of these cells between preeclamptic and non-preeclamptic women. Buemi et al (6) suggested that the number of $\mathrm{CD}_{133^{+}}$and $\mathrm{VEGF} 2^{+}$endothelial progenitor cells in the maternal circulation increased with increasing gestational age, and that women with gestational hypertension had higher levels of the latter cells when compared with healthy pregnant women.

CD34 and CD133 are expressed by a small fraction of fetal cells in the fetal circulation. However in the present study, in maternal circulation CD34 was amplified in 65 women and CD133 was amplified in 57 women by qPCR. Gene expression was evaluated by comparison with a calibration value obtained from the analysis of a set of samples from 8 healthy pregnant women selected from the study group. CD34 expression was decreased in $30(52.6 \%)$ and increased in $27(47.4 \%)$ of the 57 pregnant women, and CD133 expression was decreased in $14(28 \%)$ and increased in $36(72 \%)$ of 50 pregnant women.

Studies have demonstrated the presence of EPCs including CD34 and CD133 in different types of atherosclerosis $(34,35)$ and in allogeneic and xenogeneic aortic valve prostheses. Endothelial cell dysfunction has been suggested to be a part of a wider maternal inflammatory reaction that is responsible for the clinical syndrome of preeclampsia (36). The preeclamptic pregnancy is the most studied among pregnancy-specific complications due to its strong association stress which can result into endothelial dysfunction. Hsp70 and Hsp27 are well-known stress response proteins expressed under various pathological conditions, including trauma, and focal or global ischemia (37). The present study demonstrated that Hsp70 and Hsp27 proteins were increased in preeclamptic women. Based on these findings, the increase in EPCs correlates with the increase of Hsps, but CD34 and CD133 changes depending on preeclampsia groups. With the statistical analysis of qPCR results for CD34 and CD133, the increase in EPCs correlates with the increase of Hsps, but CD34 and CD133 changed in the preeclampsia groups.

In conclusion, the changes in CD34 and CD133 DNA in preeclamptic compared with non-preeclamptic women remain unclear. The results of this study revealed that the levels of CD34 did not change in preeclampsia; however, it is striking that the level of CD133 expression was increased in $72 \%$ of all pregnant women.

\section{Acknowledgements}

The study was funded by the Research Fund of Istanbul University (Project no: 20411 and UDP-32968).

\section{References}

1. Roberts JM and Cooper DW: Pathogenesis and genetics of preeclampsia. Lancet 357: 53-56, 2001.

2. Salonen Ros H, Lichtenstein P, Lipworth L and Cnattingius S: Genetic effects on the liability of developing pre-eclampsia and gestational hypertension. Am J Med Genet 4: 256-260, 2000.

3. Nguyen Huu S, Dubernard G, Aractingi S and Khosrotehrani K: Feto-maternal cell trafficking: A transfer of pregnancy associated progenitor cells. Stem Cell Rev 2: 111-116, 2006.

4. Gussin HA, Bischoff FZ, Hoffman R and Elias S: Endothelial precursor cells in the peripheral blood of pregnant women. J Soc Gynecol Invest 9: 357-361, 2002.

5. Sugawara J, Mitsui-Saito M, Hoshiai T, Hayashi C, Kimura Y and Okamura K: Circulating endothelial progenitor cells during human pregnancy. J Clin Endocrinol Metab 90: 1845-1848, 2005.

6. Buemi M, Allegra A, D'Anna R, et al: Concentration of circulating endothelial progenitor cells (EPC) in normal pregnancy and in pregnant women with diabetes and hypertension. Am J Obstet Gynecol 196: 68.e1-68.e6, 2007.

7. Zeybek YG, Günel T, Benian A, Aydınlı K and Kaleli S: Clinical evaluations of cell-free fetal quantities in pre-eclamptic pregnancies. J Obstet Gynaecol Res 39: 632-640, 2013.

8. Satterthwaite AB, Burn TC, Le Beau MM and Tenen DG: Structure of the gene encoding CD34, a human hematopoietic stem cell antigen. Genomics 12: 788-794, 1992

9. Davey DA and MacGillivray I: The classification and definition of the hypertensive disorders of pregnancy. Am J Obstet Gynecol 158: 892-898, 1988 .

10. Bianchi DW, Zickwolf GK, Weil GJ, Sylvester S and DeMaria MA: Male fetal progenitor cells persist in maternal blood for as long as 27 years postpartum. Proc Natl Acad Sci USA 93: 705-708, 1996.

11. Miraglia S, Godfrey W, Yin AH, et al: A novel five-transmembrane hematopoietic stem cell antigen: Isolation, characterization, and molecular cloning. Blood 90: 5013-5021, 1997.

12. Yin AH, Miraglia S, Zanjani ED, et al: AC133, a novel marker for human hematopoietic stem and progenitor cells. Blood 90: 5002-5012, 1997.

13. Ariga $\mathrm{H}$, Ohto $\mathrm{H}$, Busch MP, et al: Kinetics of fetal cellular and cell-free DNA in the maternal circulation during and after pregnancy: Implications for noninvasive prenatal diagnosis. Transfusion 41: 1524-1530, 2001

14. Luppi P, Powers RW, Verma V, Edmunds L, Plymire D and Hubel CA: Maternal circulating CD34+VEGFR-2 ${ }^{+}$and CD $133^{+}$VEGFR-2 $2^{+}$progenitor cells increase during normal pregnancy but are reduced in women with preeclampsia. Reprod Sci 17: 643-652, 2010.

15. Wilhelmi MH, Mertsching H, Wilhelmi M, Leyh R and Haverich A: Role of inflammation in allogeneic and xenogeneic heart valve degeneration: Immunohistochemical evaluation of inflammatory endothelial cell activation. J Heart Valve Dis 12: 520-526, 2003

16. Mazzone A,Epistolato MC,DeCaterina R, et al: Neoangiogenesis, T-lymphocyte infiltration, and heat shock protein-60 are biological hallmarks of an immunomediated inflammatory process in end-stage calcified aortic valve stenosis. J Am Coll Cardiol 43: 1670-1676, 2004.

17. Papp E, Nardai G, Söti C and Csermely P: Molecular chaperones, stress proteins and redox homeostasis. Biofactors 17: 249-257, 2013.

18. Molvarec A, Prohászka Z, Nagy B, Szalay J, Füst G, Karádi I and Rigó J Jr: Association of elevated serum heat-shock protein 70 concentration with transient hypertension of pregnancy, preeclampsia and superimposed preeclampsia: A case-control study. J Hum Hypertens 20: 780-786, 2006.

19. Fukushima A, Kawahara H, Isurugi C, et al: Changes in serum levels of heat shock protein 70 in preterm delivery and preeclampsia. J Obstet Gynaecol Res 31: 72-77, 2005.

20. Shin JK, Jeong YT, Jo HC, et al: Increased interaction between heat shock protein 27 and mitogen-activated protein kinase (p38 and extracellular signal-regulated kinase) in pre-eclamptic placentas. J Obstet Gynaecol Res 35; 888-894, 2009. 
21. Cunningham FG, Leveno KJ, Bloom SL, Hauth JC, Gilstrap LC III and Wenstrom KD (eds). Hypertensive disorders in pregnancy. In: Williams Obstetrics. 22nd edition. McGraw Hill, New York, pp761-808, 2005.

22. Lindheimer MD, Roberts JM and Cunningham FG (eds). Prevention of preeclampsia and eclampsia. In: Chesley's Hypertensive Disorders in Pregnancy. 3rd edition. Elsevier (Academic Press), San Diego, CA, pp213-226, 2009.

23. Majka M, Janowska-Wieczorek A, Ratajczak J, et al: Numerous growth factors, cytokines, and chemokines are secreted by human CD $34^{+}$cells, myeloblasts, erythroblasts, and megakaryoblasts and regulate normal hematopoiesis in an autocrine/paracrine manner. Blood 97: 3075-3085, 2001.

24. Peichev M, Naiyer AJ, Pereira D, et al: Expression of VEGFR-2 and $\mathrm{AC} 133$ by circulating human $\mathrm{CD} 34^{+}$cells identifies a population of functional endothelial precursors. Blood 95 952-958, 2000.

25. Jászai J, Fargeas CA, Florek M, Huttner WB and Corbeil D: Focus on molecules: Prominin-1 (CD133). Exp Eye Res 85: 585-586, 2007

26. Lo YM, Zhang J, Leung TN, Lau TK, Chang AM and Hjelm NM: Rapid clearance of fetal DNA from maternal plasma. Am J Hum Genet 64: 218-224, 1999.

27. Hahn S, Gupta AK, Troeger C, et al: Disturbances in placental immunology: Ready for therapeutic interventions? Springer Semin Immunopathol 27: 477-493, 2006.

28. Carty DM, Delles C and Dominiczak AF: Novel biomarkers for predicting preeclampsia. Trends Cardiovasc Med 18: 186-194, 2008.
29. Zhong XY, Gebhardt S, Hillermann R, Tofa KC, Holzgreve W and Hahn S: Parallel assessment of circulatory fetal DNA and corticotropin-releasing hormone mRNA in early- and late-onset preeclampsia. Clin Chem 51: 1730-1733, 2005.

30. Sifakis S, Zaravinos A, Maiz N, Spandidos DA and Nicolaides KH: First-trimester maternal plasma cell-free fetal DNA and preeclampsia. Am J Obstet Gynecol 201: 472. e1-472.e7, 2009.

31. Horinek A, Korabecna M, Panczak A, et al: Cell-free fetal DNA in maternal plasma during physiological single male pregnancies: Methodology issues and kinetics. Fetal Diagn Ther 24: 15-21, 2008.

32. Pomyje J, Zivný J, Sefc L, Plasilová M, Pytlík R and Necas E: Expression of genes regulating angiogenesis in human circulating hematopoietic cord blood $\mathrm{CD} 34^{+} / \mathrm{CD} 133^{+}$cells. Eur J Haematol 70: 143-150, 2003.

33. Matsubara K, Abe E, Matsubara Y, Kameda K and Ito M. Circulating endothelial progenitor cells during normal pregnancy and pre-eclampsia. Am J Reprod Immunol 56: 79-85, 2006.

34. Bauriedel G, Jabs A, Skowasch D, et al: Dendritic cells in neointima formation after rat carotid balloon injury. Coordinated expression with anti-apoptotic Bcl-2 and HSP47 in arterial repair. J Am Coll Cardiol 42: 930-938, 2003.

35. Banchereau J, Briere F, Caux C, et al: Immunobiology of dendritic cells. Annu Rev Immunol 18: 767-811, 2000.

36. Ekambaram P: HSP70 expression and its role in preeclamptic stress. Indian J Biochem Biophys 48: 243-255, 2011.

37. Shin JK, Jeong YT, Jo HC, et al: Increased interaction between heat shock protein 27 and mitogen-activated protein kinase (p38 and extracellular signal-regulated kinase) in pre-eclamptic placentas. J Obstet Gynaecol Res 35: 888-894, 2009. 